\title{
An OFDMA-Based Optical Access Network Architecture Exhibiting Ultra-High Capacity and Wireline-Wireless Convergence
}

\author{
K. Kanonakis, I. Tomkos, H. G. Krimmel, F. Schaich, C. Lange, E. Weis, J. Leuthold, M. Winter, S. \\ Romero, P. Kourtessis, M. Milosavljevic, I. Cano and J. Prat.
}

\begin{abstract}
This paper presents a novel optical access network architecture based on Orthogonal Frequency Division Multiple Access (OFDMA) technology and applied on a Passive Optical Network (PON) topology. In compliance with Next Generation Optical Access (NGOA) requirements, this architecture targets to outperform existing PON solutions in terms of total capacity, bandwidth allocation flexibility, number of users and network reach. Moreover, it provides the opportunity for convergence with wireless technologies and a smooth migration path from legacy access solutions like TDMA-PONs and DSL.
\end{abstract}

Index Terms-Orthogonal Frequency Division Multiplexing, OFDM, Orthogonal Frequency Division Multiple Access, OFDMA, Passive Optical Network, PON, OFDMA-PON, Next Generation Optical Access, NGOA, Wireline-Wireless Convergence.

\section{INTRODUCTION}

$\mathrm{T}$ HE demand for modernizing available applications to both residential and business customers implies that the network of the future will be faced with the challenge of transporting an increasing volume of bursty/dynamic datacentric traffic. Copper-based access solutions (Digital Subscriber Lines - DSL) as well as emerging [WiMax (Worldwide Interoperability for Microwave Access) and LTE (Long-Term Evolution)] wireless technologies come with severe limitations in both network reach and offered bandwidth per user. To cope with such constraints, the necessity of Fiber-to-the- $x$ ( $x=$ Home, Building, Curb etc.) is undisputable. However, current solutions do not seem to adequately address the stringent requirements identified regarding Next Generation Optical Access (NGOA) networks (relevant standards are expected by 2015): Peak bandwidth rates offered per user should be at least 1 Gbps, the architecture should be passive and optically transparent, supporting multi-stage splitting and demonstrating high system/infrastructure sharing (more than 128 users on a single interface) at low power consumption [1].

At the same time, wireless communication networks represent a rapidly growing market whereby new standards enable higher capacity, reliability and a larger number of supported users. Thus, convergence of optical and wireless networks is also a crucial requirement which will enable boosting network penetration and correspondingly justify OPEX (Operational Expenditures) and CAPEX (Capital Expenditures) for the network of the future.

One of the prominent technologies for offering FTTH is Passive Optical Networks (PON). Current standardized PONs based on Time Division Multiple Access (TDMA), like Ethernet PON (EPON) and Gigabit PON (GPON) as well as their recent updates (10GE-PON and 10G-PON respectively) have evolved as an access solution to provide simplicity and low operational cost [2]. However, scaling up TDMA-PONs to several tens of Gbps of aggregate capacity is extremely challenging due to the complexity of optical components and burst mode TDM receivers at such high data rates [3]. Wavelength division multiplexed PONs (WDM-PONs) have also been increasingly considered to deliver ultra high-speed services by enabling service providers to offer a dedicated wavelength straight to a home or business. To the negative side though, WDM-PONs are currently too costly to implement while they demonstrate limitations in terms of scalability and bandwidth granularity.

Orthogonal Frequency Division Multiplexing (OFDM) was originally conceived as a modulation method for achieving better transmission properties for bit streams in media displaying bandwidth limitations and non-uniform channel performance (i.e. radio, copper). It is based on the utilization of several low bit rate sub-carriers of the link carrying different QAM (Quadrature Amplitude Modulation) symbols simultaneously. In order to be orthogonal, individual subcarriers are spaced in multiples of the reciprocal symbol duration. This allows them to partially overlap (thus increasing spectral efficiency) but not interfere with one another at the sampling instants. A very interesting application of OFDM is Orthogonal Frequency Division Multiple Access (OFDMA), whereby different users are assigned to different OFDM subcarriers. Recently, OFDM/OFDMA has also been making its way into the optics world as well, with recent studies showing that OFDM/OFDMA can provide high capacity, long-reach and cost-effective operation for PONs [4], [5]. 


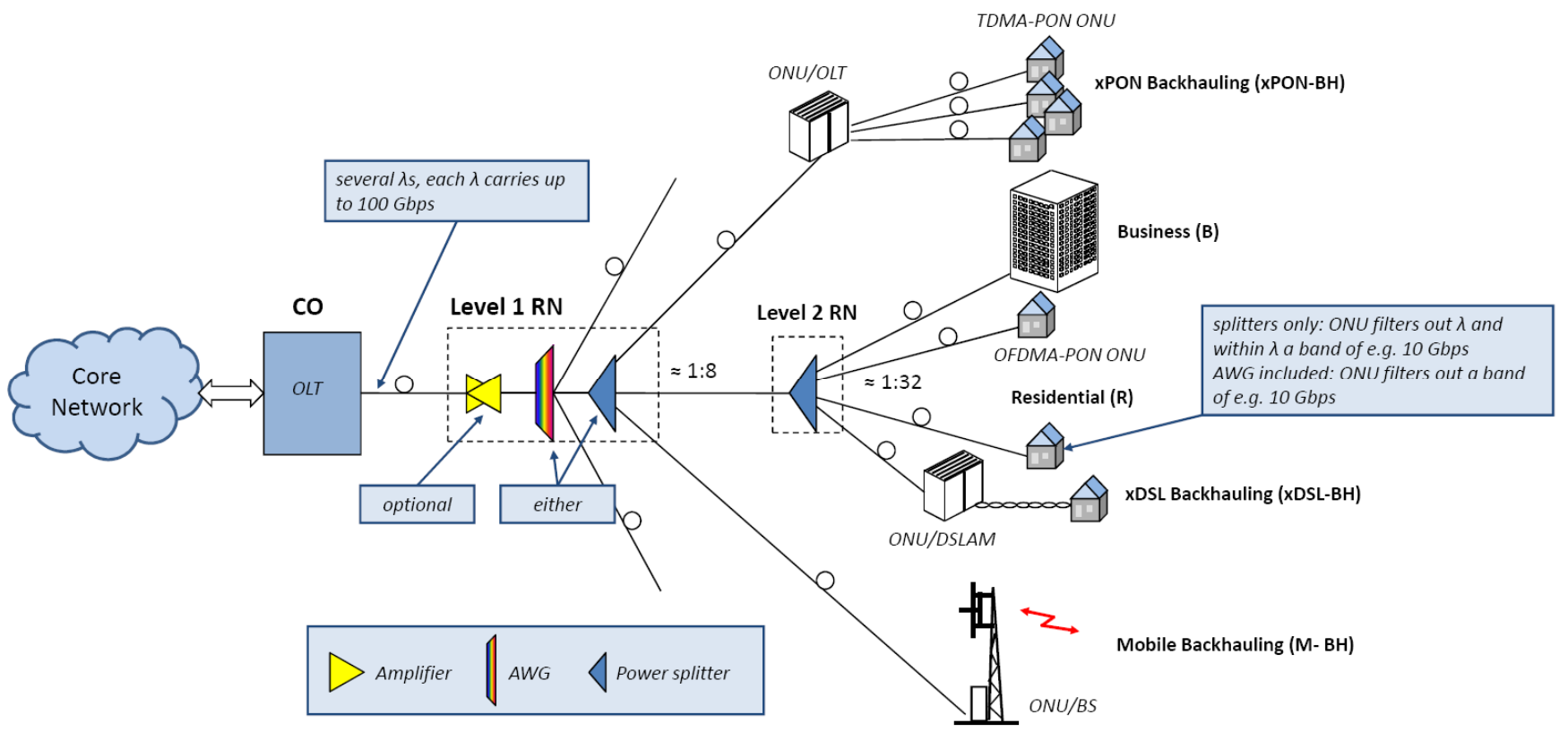

Fig. 1. Overview of the options for the ACCORDANCE network architecture and the various types of connectivity considered.

The work performed within the recently initiated EU FP7 Project ACCORDANCE targets a concept which has not been addressed until now: The introduction of OFDM/OFDMA in PONs assuming a holistic approach, i.e. addressing both the physical and the Medium Access Control (MAC) layers. Moreover, special emphasis is given to the convergence of the optical infrastructure with standard wireless solutions, as well as the migration from existing access technologies like xDSL and $\mathrm{xPON}$ ( $\mathrm{x}$ standing for their various flavors) in order to enable a seamless OFDM/OFDMA-based access network where virtually all different Telco services can be consolidated.

\section{THE ACCORDANCE ARCHITECTURE}

\section{A. Topology}

The ACCORDANCE unified topology (Fig. 1) consists of one Optical Line Termination (OLT) located at the Central Office (CO) and several Optical Network Units (ONUs). The Remote Node (RN) located at the first splitting point ("Level 1 RN") in Fig. 1, can comprise either a power coupler/splitter or a wavelength multiplexer/demultiplexer. In the first case, the advantage is that the OLT could distribute spectral segments of variable width among different services or parts of the network in a dynamic fashion, however it introduces a considerable increase in splitting loss and implies that either every single ONU in the network should have their electronics operate at the aggregate rate or that the ONUs employ optical filtering to single out specific spectral components.

The second option is to use a wavelength mux/demux (most commonly an Arrayed Waveguide Grating - AWG). This provides less flexibility in terms of segment assignment at this level (which would be static) but ensures that each ONU receives only a fraction of the total spectrum (thus processing requirements are relaxed). In addition, the use of an OFDMover-WDM approach can assist in scaling up aggregate network capacity without increasing equipment complexity/cost at the user side and at a reduced attenuation (by avoiding excessive power splitting), especially in the cases where geographical routing is obvious. Finally, a third option to further enhance user coverage and capacity, (not shown in the figure), would involve using both an AWG and splitters at each of its outputs.

The Level $1 \mathrm{RN}$ in the figure is also a candidate point in the architecture where an extender box could be placed. This introduces amplification which contrasts the purely passive nature of the PON, however it may be required in order to expand network reach when the required power budget is prohibitive.

\section{B. Node Consolidation}

One of the key benefits in the ACCORDANCE architecture is the opportunity for significant node consolidation. This suggests that operators are able to avoid using several secondary COs (e.g. sites where FTTx OLTs are currently located), thus decreasing their OPEX and also improving network reliability as well as the availability of services. Moreover, significant energy consumption reduction is expected by centralizing network equipment, for instance by reducing the need to cool outdoor equipment and by using the economies of scale for power converters. There have been three main scenarios distinguished within ACCORDANCE to address different deployment cases and offer diverse degrees of consolidation:

- Urban: This scenario suggests a completely passive access network, up to $20 \mathrm{~km}$ span length and targets densely 
populated areas.

- Passive Extended Reach:

Span lengths are up to $40 \mathrm{~km}$ with no amplification at the OutSide Plant (OSP) and the main targets in this case are network consolidation in metro-regional areas as well as wider coverage for rural areas.

- Active Extended Reach:

This scenario targets rural and special network consolidation architectures. Signal amplification is performed at the Level 1 RN site (e.g. a former local exchange building, a Digital Subscriber Line Access Multiplexer (DSLAM) cabinet or a manhole) allowing then for the maximum reach of $100 \mathrm{~km}$.

\section{Application Targets}

The proposed architecture covers a variety of "users" in need of high performance access solutions:

- Residential users:

This is the main user segment on which existing FTTH deployments are currently focused. Multi-channel high definition TV or VoD services over IP streams are offered concurrently with high speed Internet access, for a total guaranteed bit rate of up to $400 \mathrm{Mbps}$ in both directions (though in practice asymmetrical rates are still expected to be more attractive from an operator's point of view).

- Corporate/business users:

This type demands higher capacity links and has additional requirements like connection redundancy or extended security mechanisms. For many small or medium enterprises even a residential-type connection is enough, while for large corporations several tens of Gbps may be needed.

- Telco users:

This includes Telco services requesting connectivity resources to the fixed network. For example, future LTE Advanced deployments (with peak data rates in the order of multiple Gbps) will need to be supported over fibre infrastructures, since current backhauling technologies exhibit either limited capacity and moderate reach (e.g. legacy E1/T1 or symmetric DSL) or modest capacity and very short reach (VDSL2).

\section{Types of Connectivity}

The following types of connectivity are considered to be provided by the ACCORDANCE network through the ONUs:

- Ethernet Service:

This type refers to all possible network equipment that can potentially receive a similar treatment and be served by the same ONU type over the ACCORDANCE network since they share a common payload format (i.e. Ethernet frames). This category includes Residential/Corporate FTTH customers, FTTB/FTTC aggregation nodes as well as Legacy DSLAM, xPON OLTs or wireless BSs which use the ACCORDANCE network as backhaul.

- Transparent Connectivity:

In the wireless case, transparent transport implies that baseband resources of BSs are consolidated at the $\mathrm{CO}$ and connection to radio resources is achieved via the ACCORDANCE network [via Digital over Fiber (DoF) or
Radio over Fiber (RoF) techniques as will be detailed in Section IV]. Regarding transparent TDMA-PON support, more details are provided in Section VI. Finally, it is also possible to use ACCORDANCE as a means for providing transparent connectivity to already installed DSLAMs. This approach is however limited by the Signal-to-Noise Ratio (SNR) constraints due to the PON splitting (of course applicable to all transparent solutions) and the fact that this would entail reworking the existing DSL LT equipment.

\section{E. Targeted Service Clustering in ACCORDANCE}

In ACCORDANCE, each of the spectral segments mentioned in Section II.A can contain a mix of services/connectivity types depending on the needs of a certain geographical region. An example of this targeted service clustering can be seen in Fig. 2: Respective groups are created from residential/small business connectivity traffic and from mobile BS backhauling/corporate business connections, while for TDMA-PON backhauling separate groups will be required due the multi-gigabit rates per each TDMA-PON subtree respectively. Note that service selection is left to the PON terminals, which are connectivity/service-specific for cost reasons. As an example, with the proposed clustering and by accounting for the guaranteed bitrates depicted in Fig. 2(a) for the respective connectivity types per end-point (the numbers are only indicative and in addition no overbooking is considered) the overall capacity per serving area in this example is in the range of 40 to $70 \mathrm{Gbps}$. The spectral composites of those cluster capacities on a downstream optical transmitter are shown in Fig. 2(b) (subcarriers are only depicted schematically here, since the exact spectrum shape depends on the OFDM transmission scheme - see Section III). Note that cluster widths are essentially variable to allow for flexible dynamic bandwidth assignment (DBA) through the MAC layer in order to tailor the downstream spectrum to the specific needs of each service area.

\section{Physical LAyer AsPeCts}

(a)

\begin{tabular}{|l|l|l|l|}
\hline Application & User Type & Number of Users & $\begin{array}{l}\text { Guaranteed Bitrate } \\
\text { (Mbps) }\end{array}$ \\
\hline R & Home & 30 & 400 \\
\hline XDSL-BH & DSLAM & 4 & 1000 \\
\hline B & Business & 4 & 1000 \\
\hline M-BH & LTE BS & 16 & 750 \\
\hline XPON-BH & 1OG-EPON OLT & $1-4$ & 10000
\end{tabular}

(b)

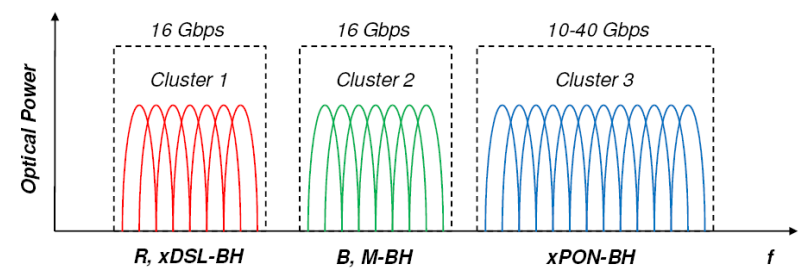

Fig. 2. (a) Application mix example for a specific geographical region and (b) the respective subcarrier spectrum breakdown, whereby service clustering has been applied. 


\section{A. Transceiver Options}

For the OFDM transmitter there are two basic options, intensity and field modulation. In the former [Fig. 3(a)], the OLT creates the groups of subcarriers in the Radio Frequency (RF) domain using OFDM, typically with an $m$-QAM modulation format. An Inverse Fast Fourier Transform (IFFT) with Hermitian symmetry is then applied to generate parallel real-valued OFDM symbols, which are serialized and then converted into an analog signal waveform using a Digital-toAnalog Converter (DAC). Then the produced signal is modulated onto the optical intensity to come up with the downstream signal [6]. In general, the entire optical spectrum must be $\mathrm{O} / \mathrm{E}$ converted at the receiver, while the different spectral segments are extracted in the electrical domain applying e.g. RF filtering and are subsequently electronically processed. This is a very important benefit compared to TDMA-PON, since it can significantly relax the electronics at the ONU side when scaling up to high (e.g. 40 Gbps) aggregate bandwidth.

Intensity Modulation (IM) OFDM is attractive due to its low cost, which makes it suitable for short-haul applications (i.e. access networks) [6]. In the case of optical field modulation there are two options: The first [Fig. 3(b)] involves upconverting the RF OFDM signal to a higher frequency by means of an RF-IQ-mixer. This RF-mixer turns the complexvalued OFDM signal into a real one. Then the signal is sent to a simple Mach-Zehnder Modulator (MZM). However, it is important to mention that the optical spectrum has two sidebands that are symmetric over the optical carrier. A narrow optical filter is used after the modulator to filter out one redundant sideband and reduce the power of the carrier [6]. The other option [Fig. 3(c)] is to use a colorless transmitter with a complex optical IQ (oIQ) modulator that comprises two MZMs, each driven by the real and imaginary part respectively of the complex electrical baseband signal for generating an optical signal [6]. By using this modulator there is no need of an optical filter to reject one of the OFDM bands since it already produces a single sideband without optical carrier [7].

Regarding OFDM signal reception, a simple Direct Detection (DD) Optical OFDM (DD-OFDM) receiver requires an optical carrier to be transmitted, reducing receiver sensitivity. Using a spectral gap between the OFDM spectrum and the optical carrier decreases the impact of intermodulation effects, however at the cost of reduced spectral efficiency. DD-OFDM leads to simpler and lower-cost ONU receivers, because a single photodiode is required for detection and there is no need of a local oscillator (LO) and polarization controllers or polarization diversity reception [7]. On the other hand, Coherent Optical (CO) OFDM provides increased spectral efficiency because it requires neither a transmitted optical carrier nor a gap between the carrier and the OFDM band.. However, its hardware implementation is more complex and costly, while it is polarization dependent and sensitive to phase noise (requiring a narrow linewidth oscillator with a low lambda offset [7]).
Initial simulations were performed in the course of the ACCORDANCE project regarding combinations of the above techniques applied to a single downstream $\lambda$ of $40 \mathrm{Gbps}$ [the main assumptions being 16-QAM, 256 generated subcarriers, 32 ONUs and a Bit Error Ratio (BER) threshold of $10^{-3} \mathrm{]}$. The results indicate that oIQ-CO transmission can achieve slightly less than $60 \mathrm{~km}$ without amplification, while for IM-DD the maximum achievable distance is restrained to roughly $30 \mathrm{~km}$.

\section{B. OLT/ONU System Design}

It is obvious that technology performance is not the sole feature to be considered regarding system design since OLTs and ONUs imply different cost requirements. The ONU, being a device to be placed at all customer premises (or all end points in general), should be as affordable as possible. Therefore, DD-OFDM seems to be the most attractive solution for the ONU Rx while the ONU Tx can employ an intensity modulation scheme based either on a direct modulated laser (DML) or a reflective solution, with the carrier seeded from the OLT or the RN. On the other hand, the OLT is shared by many customers, partitioning the total cost among them. Consequently, higher complexity solutions can be used without substantially increasing overall cost. Therefore, an optical IQ modulator is proposed for the OLT Tx, with the insertion of an artificial optical carrier for being directly detected in the ONU. For the OLT receiver CO-OFDM detection is preferable due to its enhanced sensitivity.

\section{Adaptive Modulation}

As mentioned above, OFDM is typically accompanied by the $m$-QAM modulation format. Depending on $m$ [i.e. size of the QAM constellation map and accordingly the number of bits per symbol, $\log _{2}(m)$ ], different bitrates can be achieved.

(a)

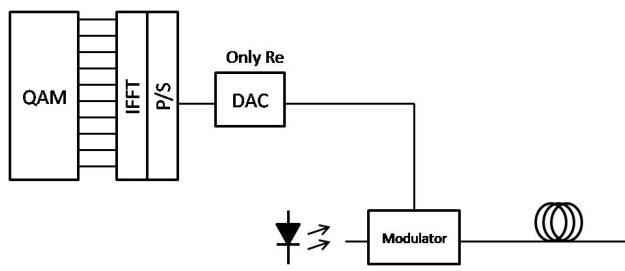

(b)

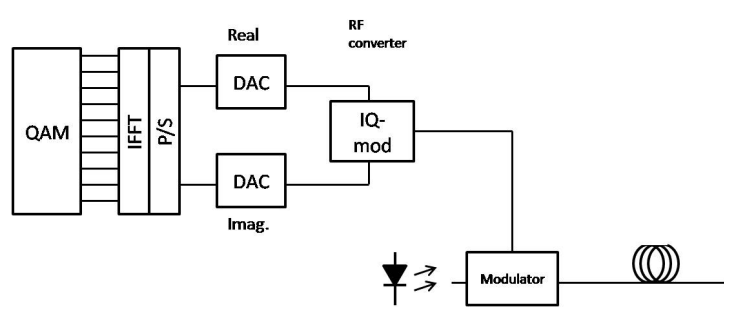

(c)

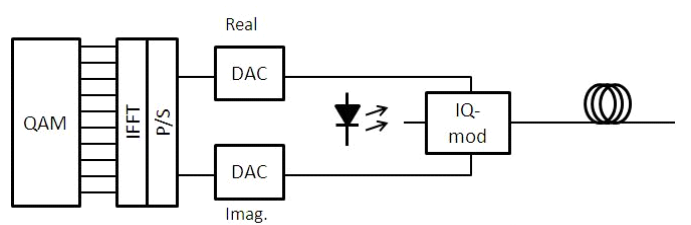

Fig. 3. OFDM Transmitter options: (a) Intensity modulation, (b) field modulation with RF up-conversion and (c) field modulation with optical IQ modulator. 
When using a fixed constellation for all subcarriers though, the subcarrier with the poorest performance determines the overall transmission performance of the system. In order to improve BER, several adaptive algorithms have been proposed which allocate optimally bits on each subcarrier according to the channel response [8]. Generally speaking, higher modulation formats are used on subcarriers exhibiting higher SNR. Similarly, in ACCORDANCE, a different QAM modulation format is used for the specific subcarriers assigned to each $\mathrm{ONU}$, depending on their transmission quality (mainly affected by varying OLT-ONU distances and splitting attenuation).

\section{WIRELINE-WIRELESS CONVERGENCE}

\section{A. Further Options for Wireless Support}

As mentioned in Section II.D, mobile BSs can be backhauled through the ACCORDANCE network (i.e. they can be thought of as normal OFDMA-PON ONUs). Perhaps the most important aspect to be taken into account in that case is that L2 control and management should be designed to realize end-to-end packet transmission adhering to the minimum performance requirements of Mobile WiMAX or LTE. However, wireless signal processing centralization can also be achieved using signal transport over the ACCORDANCE network as Analogue RoF or Digital over Fiber (DoF) radio transmission, the latter based on the Common Public Radio Interface (CPRI) [9]. Both options allow for signal transparency and independent bandwidth scheduling from the optical counterpart. When RoF is employed, WiMAX/LTE channels are shifted in frequency using a predetermined LO and Band-pass Filters (BPFs) in the OLT prior to being combined and modulated onto an optical carrier to address individual remote BSs. At the ONU/BS side, an RF-LO is required operating on the same frequency for the specific ONU/BS to downshift the wireless channels. Bandpass filters (BPFs) are needed to select each channel prior to transmission over the air. Considerable frequency guards would need to be inserted around the subcarriers used for RoF transmission, while frequency synchronisation at the ONU/BS is also challenging. On the other hand, DoF transmission with CPRI has become a prominent solution for the mobile operators to connect Remote Radio Heads (RRHs) with central units efficiently and with low deployment cost. The use of CPRI simplifies site installations (simple RRHs) since most of the digital signal processing is performed centrally. However, high data rate with very low BER is required to transport CPRI encoded WiMAX/LTE signals on the fibre. Additionally, in long-reach scenarios, high latencies could be an issue. This can be resolved by the introduction of an intermediate node between the $\mathrm{CO}$ and the remote $\mathrm{BS}$ that will be responsible for generation and transmission of CPRI streams over shorter fibre distances.

(a)

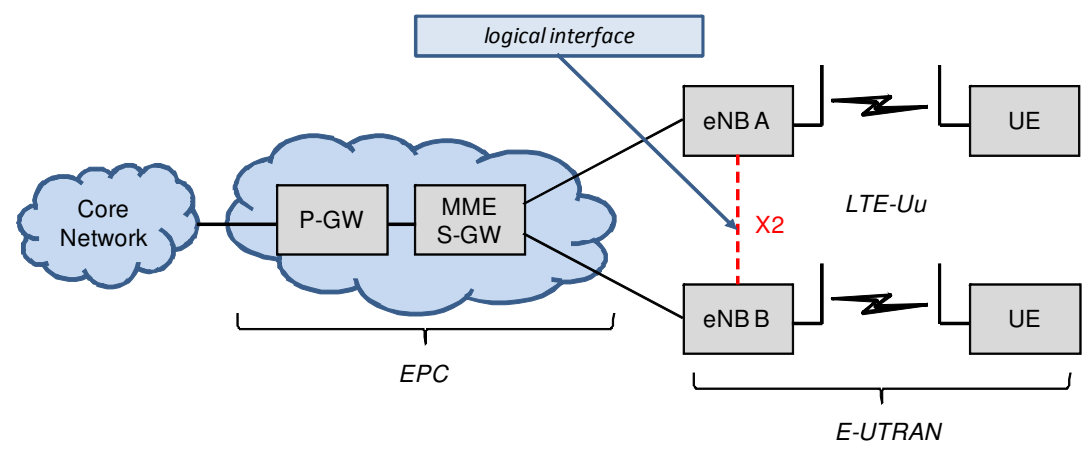

UE: User Equipment E-UTRAN: Evolved Universal Terrestrial Radio Access Network eNB:E-UTRAN Node B MME: Mobility Management Entity P-GW:PDN Gateway S-GW: Serving Gateway EPC: Evolved Packet Core

(b)

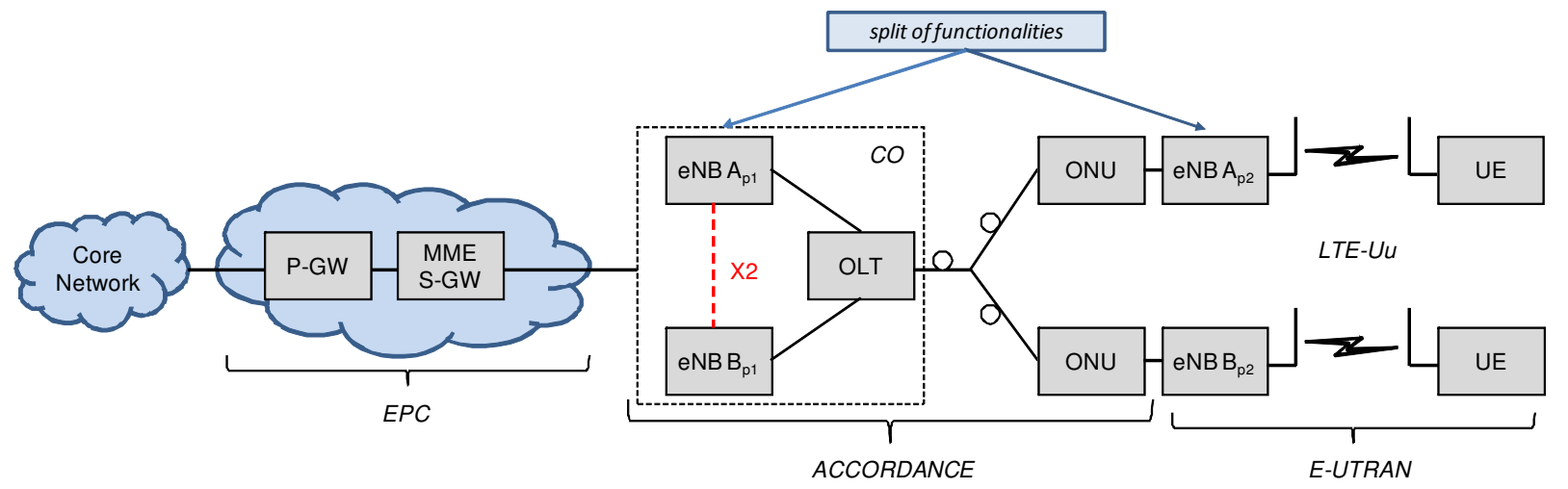

Fig. 4. (a) Overview of the basic LTE architecture and (b) its transformation in ACCORDANCE to include centralized wireless processing. 
(a)

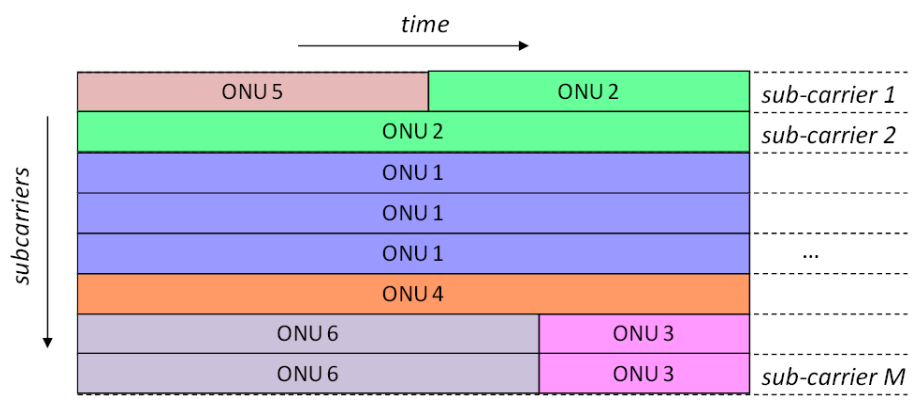

(b)

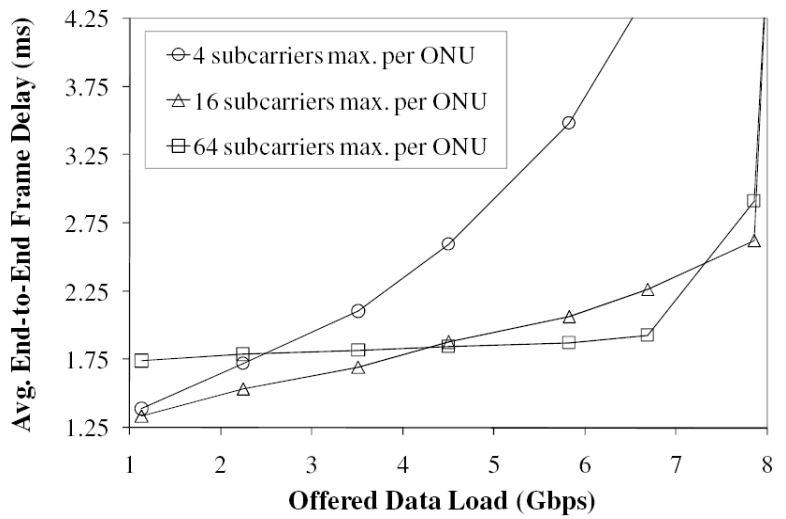

Fig. 5. (a) Hybrid OFDMA/TDMA (intra-segment) MAC operation in ACCORDANCE and (b) indicative MAC simulation results in a single segment of the ACCORDANCE architecture.

\section{B. Centralized Wireless Processing}

The centralization of ACCORDANCE is expected to enable/improve several extended wireless features like Collaborative Multi-Point (COMP) operation, self optimization and topology control, as well as reduce handover cost. For instance, Fig. 4(a) depicts the basic LTE architecture from the core to the wireless user equipment (UE). X2 is the logical interface with which the BSs are communicating with each other. Collaborative wireless schemes, such as COMP, are extensively using this channel to interchange measurements between the BSs and in some of those schemes the amount of bandwidth needed can be very high. Additionally, excessive latency due to this exchange can degrade the performance of the wireless links. In ACCORDANCE, the X2 channel is now local and virtually for free [Fig. 4(b)], however it should be taken into account that the latency between the wave generation and the reception at the UE is higher (mainly due to the fiber propagation delay), therefore the OFDMA-PON MAC should take care to keep it below guaranteed bounds.

\section{THE ACCORDANCE OFDMA-PON MAC LAYER}

A MAC protocol tailored for ACCORDANCE has to operate on two levels: The inter-segment and the intra-segment. In the first case, the spectral segments themselves must be provisioned to different service clusters (the aggregate capacity in each segment will depend on the exact service types) in a fair and dynamic way. In the latter, the ONUs belonging to each service cluster must share the subcarriers within the corresponding segment. Intra-segment bandwidth assignment is more dynamic and thus more challenging from a MAC point of view and this is the reason for being given more focus in the framework of ACCORDANCE.

Various modes of DBA are supported depending on the ONU type and requirements. The MAC protocol of ACCORDANCE is designed in such a way to provide the tools for implementing all possible options. For example, fixed subcarrier assignment is possible and in that case the ONU can use the indicated subcarrier range in a continuous manner until it is instructed otherwise. This mode is expected to be suitable for less dynamic traffic since it leads to some form of virtual circuits (particularly useful for the Mobile/xDSL/xPON backhauling scenarios). On the other hand, there is the hybrid OFDMA/TDMA mode [formation of transmission pipes, or two-dimensional "rectangles" like what is shown in Fig. 5(a)] expected to prevail for bursty traffic patterns, providing the finest granularity in network bandwidth assignment [10]. A separate channel consisting of a number of subcarriers is reserved for exchanging control information. The most important additional control fields identified in terms of bandwidth assignment are the assigned low/high subcarrier indices (which in conjunction with the start/stop times define the assigned bandwidth rectangles). In contrast to a typical PON MAC, this information must also be conveyed for the downstream transmission since ONUs should not process the entire subcarrier range.

In addition, the MAC layer must handle all procedures related to the adaptive modulation concept as explained in Section III.C. This involves the following additional functionalities:

- Introduction of a new Physical Layer Operation and Maintenance (PLOAM) downstream message for the OLT to indicate the exact $m$-QAM format to be used by the ONU.

- Monitor transmission performance (i.e. BER) by the OLT through appropriate MAC PLOAM message exchanges during the registration process (a series of them should be needed per ONU to find the optimal format for a given BER threshold).

Fig. 5(b) presents simulation results obtained within the ACCORDANCE project, assuming an EPON-based online scheduling framework, whereby each ONU can get dynamically allocated a variable number of subcarriers up to a certain value as described above (the rectangle offering the minimum delay is selected each time). A segment of $10 \mathrm{Gbps}$ is considered, split in 64 data subcarriers and serving 32 ONUs located in distances between 0 and $100 \mathrm{~km}$ from the OLT. Given the significant processing effort (at the ONU side) as well as the scheduling complexity increase (at the OLT side) when the subcarrier range increases, from this figure the optimal setting seems to be the use of roughly 16 subcarriers at maximum per ONU. 


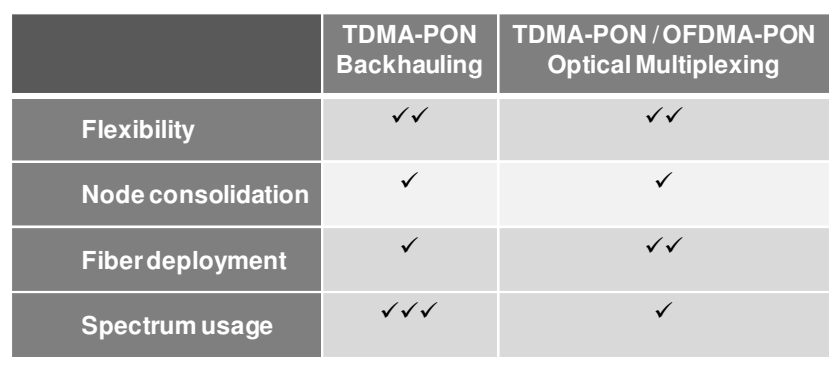

Fig. 6. Qualitative comparison of the options for coexistence with TDMAPONs.

\section{COEXISTENCE WITH TDMA-PONS}

As mentioned in Section II.D, one solution for hosting TDMAPONs is to have an OFDMA-PON ONU play the role of a TDMA-PON OLT (backhauling case). Therefore, TDMA frames are transmitted only up to a secondary $\mathrm{CO}$ where the aforementioned node is located. This ONU will of course require increased bandwidth, protection mechanisms, etc. but note that its cost would be typically shared by multiple (i.e. 1632) PON customers per port. Although this solution is quite easy to deploy in the OSP, a main downside is that the TDMAand OFDMA-PON systems will use separate PON trees, hence different fiber strands and splitter groups would be necessary for each technology, implying CAPEX and OPEX increase. In addition, only partial coexistence is offered - customers cannot be mixed in the ODN.

Another possible solution considered within ACCORDANCE is to multiplex the OFDMA-PON and TDMA-PON signals optically (transparent case). This multiplexing will take place at the $\mathrm{CO}$ (though for long-reach scenarios it might be needed to be performed at a secondary COs hosting TDMA-PON OLTs - contradicting thus the node consolidation principle of ACCORDANCE during the transition phase). This solution, though simple, requires some extra attention in case AWGs are employed in the network: Since an AWG essentially acts as an optical Band-Pass Filter (BPF), the TDMA-PON signal has to be by-passed. Besides, another drawback lies on the utilization of the optical spectrum, since TDMA-PON wavelengths and their associated frequency bands cannot be used by the OFDM system along the whole OFDMA-PON ODN. Fig. 6 provides a qualitative comparison of the aforementioned options.

\section{SUMMARY}

Before concluding, and after having presented all aspects of the proposed architecture, it would be instructive to briefly summarize the main benefits expected to emerge from ACCORDANCE.

First of all, the node consolidation philosophy which guides the whole concept allows for significant network operation benefits since a considerable number of functions are centralized. Of course, the converged nature of ACCORDANCE, addressing a wide portfolio of services and connectivity types as well as an increased number of users via the existing passive ODN infrastructure, all obviously lead to both CAPEX and OPEX reductions. This is further augmented by the fact that ACCORDANCE is a scalable solution, with the potential to offer increasingly higher capacities without the complexity burden of high-speed optics and ultra-fast hybrid burst mode operation.

Moreover, better utilization of network resources is achieved on the one hand due to the increased bandwidth granularity achieved via the use of OFDMA (as opposed to WDM solutions) and on the other as a result of the flexibility to dynamically allocate spectral segments tailored to the needs of different services and service providers (i.e. unbundling). The latter also facilitates coexistence with and migration from existing access technologies. Additionally, spectral resource usage benefits result both from the increased spectral efficiency offered by OFDM, as well as by the possibility for dynamically adjusting the subcarrier modulation formats.

Finally, although at first glance OFDM processing is more complex and thus more energy-demanding than for instance simple Non-Return-to-Zero (NRZ) transmission, it can be argued that ACCORDANCE inherently provides energyefficient network operation: First of all, it can essentially operate over a fully passive OSP infrastructure, while node consolidation and convergence allow skipping many intermediate high-speed interfaces and thus saving the associated electrical power consumption.

\section{CONCLUSION}

This work has presented in detail a novel access network paradigm (ACCORDANCE) which offers ultra-high data rates, enhanced reach and flexibility, as well as convergence of multiple types of connectivity by leveraging on OFDMA technology in a typical PON topology. The challenges associated with each aspect of such a network have been detailed and solutions have been proposed.

\section{ACKNOWLEDGMENTS}

This work was supported in part by the EU FP7 STREP ACCORDANCE (http://www.ict-accordance.eu/).

\section{REFERENCES}

[1] D. Breuer, R. Hülsermann, C. Lange. T. Monath and E. Weis, "Architectural Options and Challenges for Next Generation Optical Access," Proc. ECOC'10,Turin, Italy, paper Mo.2.B.1.

[2] J. Kani et al., "Next Generation PON - part I: Technology roadmap and general requirements," IEEE Commun. Mag., vol. 47, no. 11, Nov. 2009 , pp. 43 - 49.

[3] F. Effenberger, H. Mukai, S. Park, and T. Pfeiffer, "Next-generation PON - part II: Candidate systems for next-generation PON," IEEE Commun. Mag., vol. 47, no. 11, Nov. 2009, pp. 50-57.

[4] N. Cvijetic and D. Qian and J. Hu, "100 Gb/s Optical Access Based on Optical Orthogonal Frequency-Division Multiplexing," IEEE Commun. Mag., vol. 48, no. 7, Jul. 2010, pp. 70-77.

[5] C. W. Chow et al., "Demonstration of High Spectral Efficient OFDMQAM Long Reach Passive Optical Network," Proc. ECOC'08, Brussels, Belgium, paper Th.2.F.5.

[6] B. J. C. Schmidt, A. J. Lowery, and J. Armstrong, "Experimental Demonstrations of Electronic Dispersion Compensation for Long-Haul Transmission Using Direct-Detection Optical OFDM," J. Lightwave Technol., vol. 26, no.1, Jan. 2008, pp. 196-203. 
[7] D. Qian, N. Cvijetic, J. Hu, T. Wang, "Optical OFDM transmission in metro/access networks," Proc. OFC'09, San Diego, CA, paper OMV1.

[8] J. M. Tang, K. A. Shore, "Maximizing the transmission performance of adaptively modulated optical OFDM signals in multimode-fibre links by optimizing analog-to-digital converters," J. Lightwave Technol., vol.25, no.3, Mar. 2007, pp.787-798.

[9] Common Public Radio Interface (CPRI); Interface Specification V4.1, 2009-02-18.

[10] K. Kanonakis, E. Giacoumidis, I. Tomkos, "Physical Layer Aware MAC Schemes for Dynamic Subcarrier Assignment in OFDMA-PON

Networks," J. Lightwave Technol., 2012, doi: 10.1109/JLT.2012.2187349.

Konstantinos Kanonakis (kkan@ait.gr) was awarded his PhD and Dipl.-Ing degrees in 2007 and 2004 respectively, both from the National Technical University of Athens (NTUA), Greece. His main research interests are in the area of architectures and control protocols for broadband access and optical core networks. He has co-authored more than 40 papers that appeared in international peer-reviewed journals and conferences and has participated in several EU-funded projects.

Ioannis Tomkos (itom@ait.gr) is with AIT, Greece. He was/is involved with leading role in many European and national research projects. Together with his colleagues and students he has co-authored over 420 peer-reviewed journal/conference articles. Dr. Tomkos is involved in the editorial boards of many scientific journals and in the organizing committees of numerous conferences.

Heinz-George Krimmel (Heinz-Georg.Krimmel@alcatel-lucent.com) received the Dipl.Ing. degree in electrical engineering from the University of Stuttgart, Stuttgart, Germany. In 1987, he joined the Alcatel Research Center and since then is active in the access networks domain in various fields. He is currently with the Optical Access Group, Alcatel-Lucent Bell Labs, Stuttgart, Germany.

Frank Schaich (frank.schaich@alcatel-lucent.com) has worked from 2002 to 2007 as research assistant at the Institute of Communications (University of Stuttgart) and received in 2008 the Dr.-Ing. degree. He joined Alcatel-Lucent in 2007. Currently his focus is on cost-efficient architectures for Centralized Radio Access Networks (CRAN) and on the definition of cellular networks beyond LTE-advanced.

Christoph Lange (Christoph.Lange@telekom.de) received the Dipl.-Ing degree (diploma) in electrical engineering and the Dr.-Ing. degree (Ph. D.) in communications engineering from the University of Rostock, Germany, in 1998 and 2003, respectively. Currently, he is with Deutsche Telekom, Telekom Innovation Laboratories, Berlin. His work is focused on broadband access networks and on the energy efficiency of telecommunication networks.

Erik Weis (erik.weis@telekom.de) received his Dipl.-Ing. degree in electrical engineering from the Technical University of Dresden. Since joining Deutsche Telekom AG in 1997 he was mainly concerned with the development of access network evolution strategies. Since 2009 he is with Deutsche Telekom Laboratories. His current research interests include nextgeneration access networks and network convergence.

Juerg Leuthold (Juerg.Leuthold@kit.edu) has a Ph.D. degree in physics from the Swiss Federal Institute of Technology (ETH) Zuerich. He is a full Professor at the Karlsruhe Institute of Technology (KIT), head of the Institute of Photonics and Quantum Electronics (IPQ) and director of the Helmholtz Research Association Institute of Microstructure Technology (IMT) at KIT.

Marcus Winter (marcus.winter@kit.edu) is with the Institute of Photonics and Quantum Electronics (IPQ) of the Karlsruhe Institute of Technology (KIT).

Sergio Romero (srg@tid.es) received his M.S. degree in telecommunication engineering from the University of Zaragoza. With Telefónica R\&D since 2003 he has been involved and managed several internal and European collaborative projects (e.g. FP6-MUSE) related to broadband access networks. His expertise spans from copper and fiber technologies to mobile backhauling.
Pandelis Kourtessis (p.kourtessis@ herts.ac.uk) completed his PhD at Essex University UK. He joined the University of Hertfordshire, UK, in October 2003 where he led the activities of the Optical Networks Research Group. His work focuses at Next-Generation Passive Optical Networks, including wireless backhauling, and the development of XGPON MAC protocols.

Milos Milosavljevic (m.milosavljevic@herts.ac.uk) received his BEng, MSc and $\mathrm{PhD}$ degrees from University of Hertfordshire where he is currently working as a post-doctorate researcher on optical and wireless integration. His current research interest include WiMAX/LTE convergence with PONs, next generation optical networks, radio over fibre $(\mathrm{RoF})$ and digital system design.

Ivan N. Cano (ivann.cano@gmail.com) received the PhD degree from Scuola Interpolitecnica (Politecnico di Torino) in 2010. He is currently sponsored by Mexican council CONACYT as a Post-Doctoral researcher in Universitat Politecnica de Catalunya (UPC). He has participated in several European Projects and has experience in the telecommunication industry.

Josep Prat (jprat@tsc.upc.edu) is full professor in the Optical Communications Group of the Signal Theory and Communications Department in the Universitat Politècnica de Catalunya. He has led the European FP7 project SARDANA on next-generation FTTH access networks, and has participated in several EU projects on optical transport and access networks. 